\title{
Evaluación de aptitud de tierras al cultivo de caña de azúcar en la Huasteca potosina, México, por técnicas geomáticas ${ }^{1}$
}

\author{
Noé Aguilar Rivera², Luis Alberto Olvera Vargas ${ }^{3}$ \\ y Guadalupe Galindo Mendoza ${ }^{4}$
}

\begin{abstract}
RESUMEN
En la competitividad de la industria azucarera mexicana se requiere la reestructuración del proceso productivo, planificar y optimizar el uso de recursos técnicos, financieros y humanos. En la Huasteca, México, el área cañera está incrementándose para la producción de azúcar y bioenergía debido a su rápido crecimiento y producción de energía por hectárea. Sin embargo, los rendimientos de caña de azúcar/ha han disminuido por la gran demanda de nutrientes del suelo que son removidos con el cultivo continuo. Esta investigación se llevó a cabo para analizar el efecto de la producción continua de caña de azúcar comercial en las propiedades químicas del suelo usando datos de las zonas productoras de la Huasteca y la integración de técnicas de agricultura de precisión (AP), gestión de suelos y evaluación de tierras. La disminución de la materia orgánica bajo cultivo continuo confirmó su dependencia con el rendimiento de caña de azúcar y la susceptibilidad de los suelos a la influencia del medio ambiente
\end{abstract}

Palabras clave: Evaluación de Tierras, caña de azúcar, Sistemas de Información Geográfica

\begin{abstract}
Mexican sugarcane industry competitiveness needs to reestructuring its productive process in order to planning and optimizes the use of the technical, financial and human resources. At Huasteca Mexico an increasing area of sugarcane is being growing for the production of sugar and bioenergy because of its rapid growth and high energy production per ha. However, sugar cane yields had declined because sugarcane crop puts a high demands on the soil due to large amounts of nutrients are removed with the harvest. This research was carried out to analyze the effect of commercial sugarcane production on soil chemical properties using data from the main producing areas from Huasteca and integrating the approaches as Precision Agriculture (PA), soils management and Land Evaluation. The decrease in soil organic matter under continuous sugarcane confirmed dependence of cane yield and increases the susceptibility of the sugarcane soils to influence of the environment
\end{abstract}

Key words: Land Evaluation, sugarcane, GIS

1 Artículo recibido el 20 de enero de 2012, aceptado el 16 de agosto de 2012 y corregido el 21 de enero de 2013.

2 Facultad de Ciencias Biológicas y Agropecuarias, Universidad Veracruzana (México).

E-mail: naguilar@uv.mx
3 Laboratorio Nacional de Geoprocesamiento de Información Fitosanitaria (LaNGIF). Universidad Autónoma de San Luis de Potosí (México).

Email: olvera@uaslp.mx

4 Laboratorio Nacional de Geoprocesamiento de Información Fitosanitaria (LaNGIF). Universidad Autónoma de San Luis de Potosí (México).

E-mail: ggm@uaslp.mx 
En México, el cultivo de caña de azúcar (Saccharum spp.) es una actividad agrícola de relevancia económica y se extiende por distintas regiones agroecológicas. Cada una de ellas posee características fisiográficas, climáticas y edáficas particulares y otros factores limitantes complejos, lo que genera diferentes condiciones y aptitudes para el cultivo. Se produce en 227 municipios ubicados en 15 estados: Veracruz, Jalisco, San Luis Potosí, Tamaulipas, Oaxaca, Sinaloa, Chiapas, Tabasco, Campeche, Quintana Roo, Michoacán, Colima, Puebla, Nayarit y Morelos en los cuales se concentra el $13 \%$ de la población nacional (más de 3 millones de personas dependen directa e indirectamente de su cultivo y transformación); genera el $0.5 \%$ del producto interno bruto (PIB) nacional: $11.6 \%$ del PIB del sector agropecuario, el $2.5 \%$ de la industria manufacturera. México es el séptimo productor mundial de azúcar y en la zafra 20102011 la superficie cosechada fue de 673,050 ha, con una producción total de caña molida de 42.529.222 t, en 57 ingenios azucareros con una producción azucarera de 5,183,500 $\mathrm{t}$, con rendimientos de fábrica $11.75 \%$, de campo de $65.6 \mathrm{t}$ caña ha-1 (TCH) y agroindustrial 7.7 t sacarosa ha-1 (TSH) que han presentado en la presente década una drástica disminución de la productividad de campo (Aguilar et al., 2012).

En este sentido, la toma de decisiones en la agroindustria azucarera mexicana necesita prever el comportamiento de su proceso productivo en campo, con el fin de planificar y optimizar el uso de los recursos técnicos, humanos y financieros. Sin embargo, la operatividad, competitividad y contribución al impulso de la capacidad fabril para su molienda, presenta enormes desafíos emanados de la histórica crisis estructural y múltiples contradicciones coyunturales aún presentes en el sector cañero mexicano, con consecuencias negativas tales como: infraestructura y tecnología agroproductiva poco eficientes, elevados costos de producción y uso exiguo de los productos y subproductos obtenidos en el ingenio azucarero, trapiche y destilerías, sin desestimar la capacidad organizativa de los productores e insuficiente planificación operativa de la siembra, labores culturales y la cosecha y recolección (corte, alza, transportación y recepción) de la caña de azúcar
Herrera et al. (2009) y Romero et al. (2009) reportaron que la caña de azúcar es una de las plantas de más altos rendimientos en biomasa por área y unidad de tiempo y produce sacarosa, que es el alimento energético de consumo humano más difundido en el mundo, una parte de sus necesidades de fertilizantes, la energía necesaria para su elaboración industrial y es materia prima de alrededor de un centenar de productos derivados de gran valor para el desarrollo humano. Por cada tonelada de tallos molidos, en el proceso de transformación agroindustrial, se extraen $250 \mathrm{~kg}$ de bagazo, $6 \mathrm{~kg}$ de cenizas, $45 \mathrm{~kg}$ de melaza, $30 \mathrm{~kg}$ de cachaza (lodo de filtros) y $14 \mathrm{~L}$ de vinaza por cada litro de alcohol producido a partir de melaza. A estas cualidades excepcionales se suma su adaptabilidad a condiciones adversas del medio ambiente, es resistente a plagas y enfermedades, y alta fijación de $\mathrm{CO}_{2}$ (hasta 80 toneladas de $\mathrm{CO}_{2}$ ), comparable a la de los bosques tropicales, características que la convierten en un cultivo productor de biomasa de gran robustez, paradigma de una agricultura sostenible, si es manejado adecuadamente por el hombre (Salgado et al., 2001).

\section{Suelos de la agroindustria cañera en México}

Las distintas características y propiedades del recurso edáfico, como el sostén físico y químico de la agricultura cañera, pueden ser determinantes de potenciales productivos diferenciales, y afectar los costos de producción, ordenamiento territorial con fines de expansión de la frontera agrícola actual y conservación, en el contexto de cambio climático y variabilidad espacial (Thorburn et al.,2012; Panosso et al., 2011 ) para que técnicos y productores cañeros tomen en consideración, para decidir la adopción de tecnologías de manejo y gestión más apropiada para los diversos factores limitantes como el estrés hídrico (Barbosa Queiroz et al., 2011; Ghiberto et al., 2011) y llevar a cabo una serie de recomendaciones que se consideran apropiadas para las características fisiográficas, edafo-climáticas y espacio temporales de cada región (Huang et al., 2011) como la aplicación de abonos orgánicos con subproductos azucareros como las vinazas y compostas (Jiang et al., 2012). 
En este contexto, Sánchez-Hernández et al. (2011), Quiroz et al. (2011) y Cotler (2003) señalaron que en México no se han desarrollado suficientes investigaciones acerca de la influencia de las diferentes formas de uso del suelo, sobre la fertilidad del mismo y manejo de la materia orgánica, lo cual ha sido abordado tradicionalmente mediante estudios edafológicos convencionales de muestreo (estadística clásica), que ha dado una solución lo más aceptable posible desde el punto de vista técnico. No obstante la precisión de los datos y la imposibilidad de realizar análisis espacial (ordenamiento territorial y análisis geoestadístico), se ha visto reflejada en la aplicación no siempre correcta de los criterios económicos y un manejo individual de los insumos o medidas para el incremento de los rendimientos, como son el empleo de los fertilizantes, el riego, los abonos orgánicos, etc. mediante la manipulación, almacenamiento y procesamiento de grandes cantidades de datos, para la toma de decisiones ambientales, económicas y sociales. El uso del suelo influye sobre las propiedades químicas, físicas y biológicas, el estado de su fertilidad y la disminución de su capacidad de almacenamiento de carbono. Por lo tanto en suelos cañeros con diferentes sistemas de manejo, es necesario identificar variables y factores que influyen en su fertilidad.

La situación presente hoy en la agricultura de la mayoría de los ingenios azucareros, como en el estado de San Luis Potosí, carece de los fundamentos científicos y técnicos que sustenten una adecuada tecnología de producción y se caracterizan por bajos rendimientos y productividad, falta de orientación para la aplicación de agroquímicos, desconocimiento de los factores edáficos limitantes y deterioro ambiental (principalmente del suelo), además de elevados gastos en operación e insumos. Por lo general, se utiliza una sola fórmula de fertilizantes (o muy pocas) por ingenio; el uso de plaguicidas es indiscriminado; se aplica riego sin tener en cuenta las propiedades del suelo y exigencias del cultivo por etapa fenológica de desarrollo y levantamiento e interpretación de mapas y fotografías aéreas.

El mapa de suelo que se dispone (escala 1:250 000) es a nivel de país, muy general, sin elementos de clasificación, potencialida- des y limitaciones existentes de los suelos, identificación de unidades cartográficas y perfiles agrológicos, variabilidad espacial de suelos, estudios etno-edafológicos o clasificación campesina de tierras, datos filtrados, tabulados y actualizados de catastro rural, cartografía, caracterización, morfología, propiedades físicas y químicas, distribución de suelos y factores limitantes edafológicas para la producción y establecimiento de relaciones suelo-rendimiento, así como muchas falencias en lo relativo a normativa, metodología, calidad y aplicabilidad a mapas de aptitud agroecológica para caña de azúcar a diversas escalas (ejido, predio o región) con el fin de establecer dosis de fertilización en diferentes tipos de suelo (Gómez et al., 2010)

\section{Crecimiento del cultivo de caña de azúcar y productividad en la Huasteca, San Luis Potosí}

Este cultivo fue introducido a la zona en el siglo XVI por los españoles pero el desarrollo industrial azucarero, en la denominada región Huasteca, inicia sistemáticamente en el siglo XIX en 1888, cuando se estableció el primer ingenio azucarero denominado "Compañía Manufacturera y Desarrolladora Rascón" el cual posteriormente se transformó en The Rio Tamasopo Sugar Company Agua Buena o "Ingenio Agua Buena" (1903) hasta su reestructuración en "Ingenio Alianza Popular" en 1975 (Aguilar, 2010).

La actividad ingresa en un proceso acelerado de modernización y expansión de la superficie cañera por la creación del Ingenio Plan de Ayala en 1963, en la planicie de Ciudad Valles y El Naranjo, y en el norte del municipio de Aquismón. El cultivo de caña de azúcar tuvo un punto de inflexión más marcado en las décadas de los setenta y ochenta con la construcción de los ingenios Plan de Ayala (1966), Ponciano Arriaga (hoy San Miguel El Naranjo) (1975) y Plan de San Luis (1984) como parte de la política azucarera, donde el Estado asumió el papel de "promotor" del proceso económico a través del estímulo al sector privado mediante políticas de carácter proteccionista y la aplicación del modelo de "Sustitución de Importaciones" para que la industria azucarera pudiera abocarse más eficazmente a la resolución de los 
Figura $\mathrm{N}^{\circ} 1$

Desarrollo histórico y productividad de la agroindustria azucarera de la Huasteca potosina periodo 1937-2011

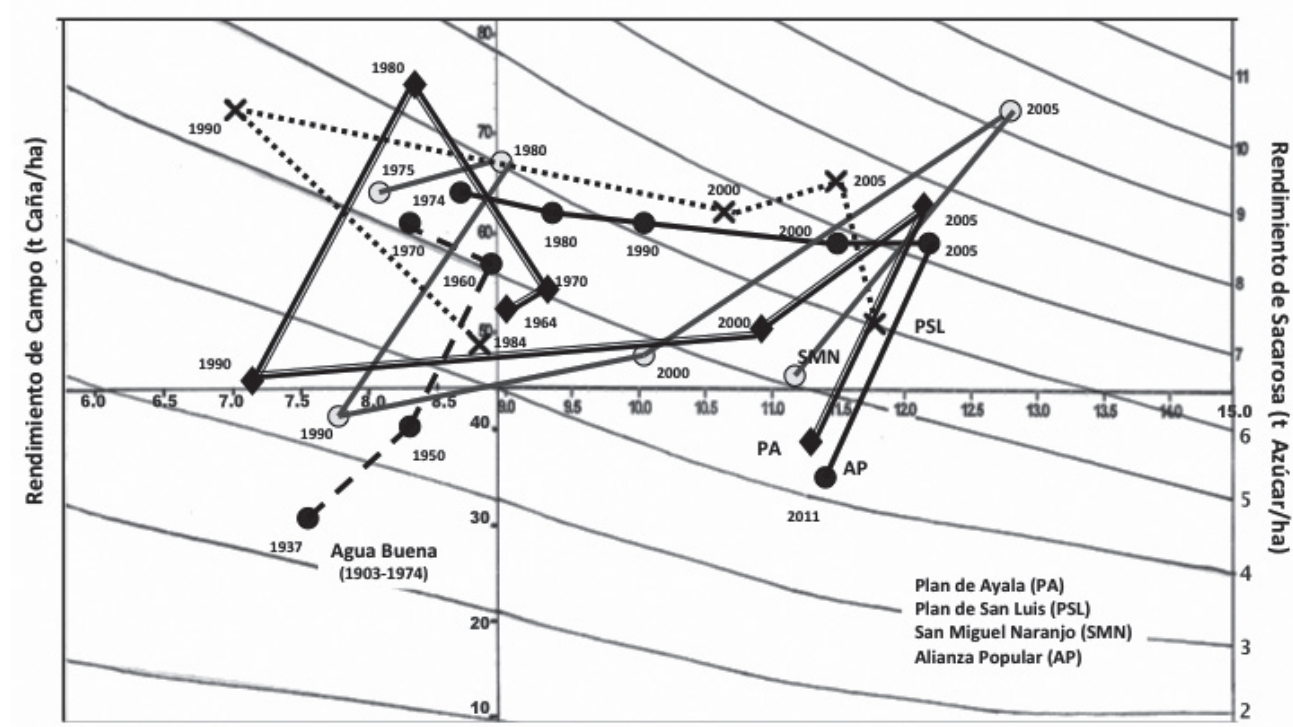

Rendimiento de Fábrica (\%)

Fuente: Elaboración propia en base a Cámara Nacional de las Industrias Azucarera y Alcoholera, Manual Azucarero Mexicano a 53 años 1958 a 2011, México D.F.

Figura $N^{\circ} 2$

Productividad y crecimiento de la superficie cañera (1937-2010)

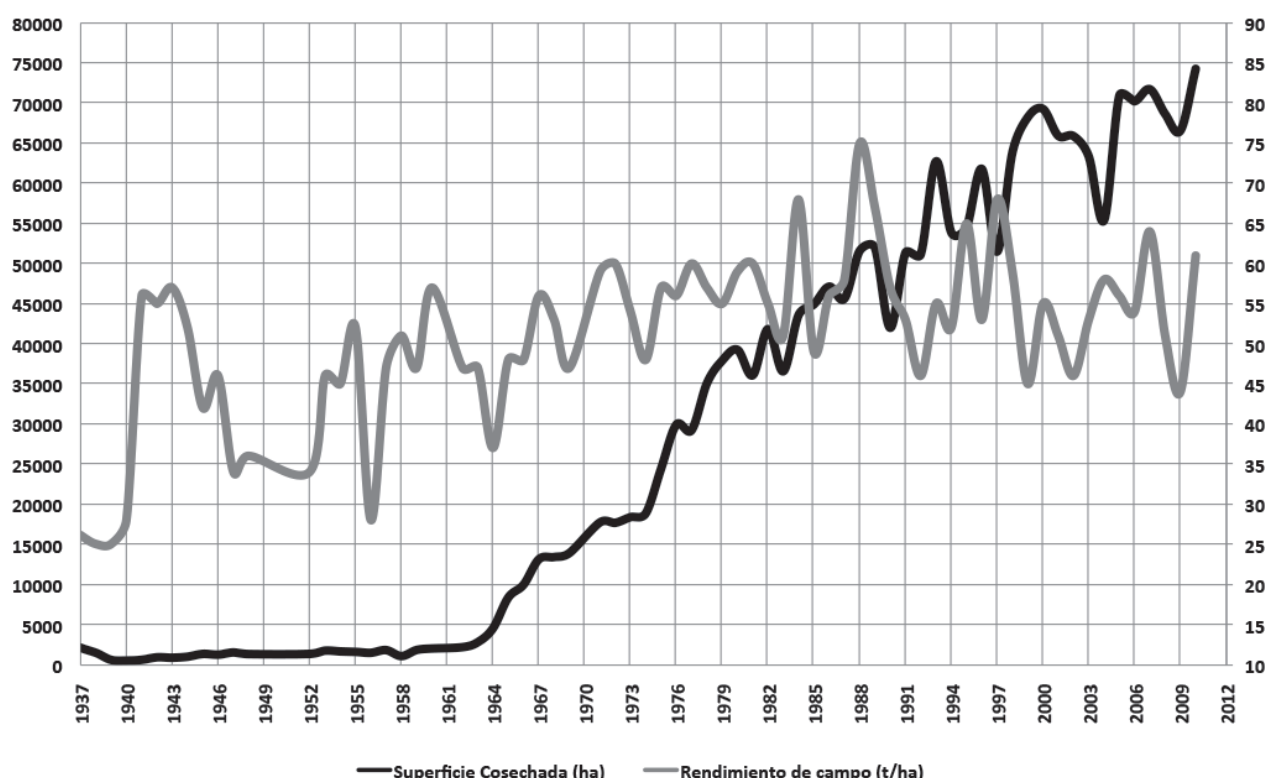

Fuente: Elaboración propia en base a Cámara Nacional de las Industrias Azucarera y Alcoholera, Manual Azucarero Mexicano a 53 años 1958 a 2011, México D.F. 
problemas tradicionales, como lo eran la relocalización de ingenios azucareros, la renovación y modernización del equipo industrial y agrícola, y el aumento de la producción en fábrica y en campo; bajo este esquema se promovería el objetivo de que el cultivo de caña de azúcar se realizara de forma intensiva y no extensiva principalmente en los municipios de Tamasopo, Ciudad Valles y el Naranjo para después extenderse a otros municipios. La producción azucarera satisfaría la demanda de los mercados nacional y extranjero. Este proyecto solo se generalizó en la Huasteca en la forma de incrementos constantes (crecimiento extensivo) en la superficie cañera y producción de azúcar en los cuatro ingenios a pesar de tener indicadores productivos por debajo de la media nacional desde sus inicios (Paré, 1987) (Figuras $\mathrm{N}^{\circ} 1$ y N²).

Por lo que para esta zona cañera, son necesarias nuevas bases paradigmáticas como la agricultura de precisión, en función de la variabilidad de los elementos de fertilidad del suelo como el nitrógeno (Portz et al., 2012), mediante el uso combinado de imágenes de satélite multitemporales y multiespectrales (Pellegrino, 2012; Patil et al., 2012; Marques Genú, 2011), sistemas de información geográfica, modelos digitales de elevación, sensores remotos, mapas analógicos y/o digitales, datos meteorológicos, visitas a campo y muestreos (Miphokasap et al., 2012); así como para la generación de información y mapeo de parámetros ambientales y socioeconómicos, debido a los diversos factores que actúan en varias escalas espaciales y temporales, naturales o antrópicas para mejorar la fertilidad del suelo y, con ello, la productividad agrícola de las zonas cañeras para ser competitivas y sostenibles (Palaniswami et al., 2011).

En este sentido, para la evaluación de tierras, es importante conocer la geología, tipo de suelos, fisiografía, clima, vegetación, variabilidad espacial y el uso de la tierra, los cuales pueden ser mapeados para propósitos específicos como la zonificación edafológica, como metodología de diagnóstico y de toma de decisiones, que proporciona las bases para la estimación de los rendimientos potenciales, evaluación de aptitud de tierras y los requerimientos para el manejo y gestión que tienen relación con la cantidad y calidad de la cosecha, el desarrollo fotosintético del cultivo de caña de azúcar, el crecimiento y el rendimiento (Aguilar, 2010; Quintero, 2008).

El uso combinado de sistemas de información geográfica (SIG), modelos digitales de elevación (DEM) y sensores remotos o teledetección juega un rol importante en el análisis exhaustivo de estos recursos naturales en la detección (directa o indirecta), mapeado, extrapolación, interpretación, cálculo de áreas y monitoreo (Patil et al., 2012; Chuvieco, 1996).

Un SIG permite la entrada, edición, almacenaje, selección, transformación, análisis, despliegue o visualización e impresión de datos espaciales. SIG son herramientas indispensables para el análisis y la presentación de mapas en la evaluación de tierras, para establecer metodologías de regionalización, zonificación y lotificación del cultivo de caña de azúcar facilitando el almacenaje y análisis de un amplio rango de datos espaciales en agricultura de precisión cañera (Pellegrino, 2012; Marques-Genú, 2011; Ruiz et al., 2011; Galdámez, 2007; Henríquez et al., 2005 y Astier et al., 2002).

La toma de decisiones con Evaluación Multicriterio (EMC) en un SIG, en gestión o gerenciamiento del cultivo de caña de azúcar, es un proceso basado en un conjunto de conceptos, modelos y métodos para describir, evaluar, jerarquizar, elegir o rechazar alternativas, con base en una valoración expresada por intensidades de preferencia, de acuerdo con diversos criterios es decir, tiene como propósito investigar un número de alternativas mediante criterios múltiples con la finalidad de auxiliar a los tomadores de decisiones a describir, evaluar, ordenar, jerarquizar, seleccionar o rechazar alternativas de uso (Ceballos-Silva, 2010).

\section{Materiales y métodos}

La Huasteca potosina pertenece al estado de San Luis Potosí, México, comprende dos provincias fisiográficas, la Sierra Madre Oriental y la Llanura Costera del Golfo Norte; por lo tanto, en el 35\% de la cuenca los terrenos son de serranía, el $30 \%$ de tipo Ilanura, el $16 \%$ a bajadas y el resto a lomeríos, 
valles y mesetas. Resultado de la conjunción de estos factores, manifiesta una significativa variedad de ecosistemas holoárticos y neotropicales. Estas características físicas han permitido el desarrollo de actividades agropecuarias, sobre todo la caña de azúcar en los municipios productores como zonas de abastecimiento cañero de cuatro ingenios azucareros presentes: Alianza Popular, Plan de Ayala, San Miguel El Naranjo y Plan de San Luis (Galindo, 2009).

Los suelos predominantes son del tipo Vertisol Pelico con el predominio de pendientes de 0 a $20 \%$ de tipo llano o casi llano a moderadamente escaparpado. El clima dominante es (A) $\mathrm{Cm}$ (Semicálido húmedo con temperatura media anual de 18 a $22{ }^{\circ} \mathrm{C}$ ), Aw1 (muy cálido subhúmedo con temperatura media mayor de $18^{\circ} \mathrm{C}$ ) y Am (cálido húmedo con temperatura media anual de 18 a $22{ }^{\circ} \mathrm{C}$ ). El tamaño de explotación promedio es 4 ha en una superficie cosechada de 62.582 ha en 12.910 unidades productivas y 12,567 productores de los cuales $72 \%$ es régimen Ejidal y $28 \%$ Privado. Las principales variedades de caña son CP 72-2086 (21\%), y ZMEX 55-32 $(50.6 \%)$ en ciclos plantilla $(13,96 \%)$, soca $(13,76 \%)$ y resoca $(45,44 \%)$ con tan solo el $27,8 \%$ con algún sistema de riego.

La Huasteca se ha ubicado a nivel nacional entre los primeros 3 productores de este cultivo y de azúcar $(10 \%$ de la producción de caña y azúcar de México) y dentro de los estados de menor rendimiento de campo y agroindustrial con un promedio anual de 56 t/ha, el cual disminuyó para la zafra 2008-2009 a 51 t/ha y 44 t/ha para la zafra 2009/2010, lo cual establece que el campo presenta fragilidad ambiental, está fragmentado y desintegrado (minifundio), improductivo y sobreexplotado; caracterizado por una agricultura de supervivencia e inercia basado en la capacidad de adaptación de la caña de azúcar y vulnerabilidad a los cambios del entorno (plagas, enfermedades, sequías etc.), derivado de cultivos estresados, sin renovación varietal y prácticas innovadoras y los ingenios azucareros (certificados en sus procesos) no muestran su potencial productivo debido a la baja calidad y abasto de materia que inclusive se cultiva por debajo del umbral económico (40 t/ha) al requerirse de 0,125-0,20 ha/t.azúcar a diferencia de la media nacional (0,09-0,11 ha/t.azúcar) (Aguilar et al., 2010).

La dosis de fertilización de N, P2O5 y K2O para caña de azúcar, cultivada en diferentes tipos de suelo, se aplica sin un modelo conceptual en el balance entre la demanda del nutrimento por el cultivo, el suministro que hace el suelo de este y la eficiencia del fertilizante basado en la interpretación de fotografías aéreas, recorridos de campo y barrenaciones en cada unidad para describir perfiles agrológicos y análisis físicos y químicos para clasificar el suelo según el Referencial Mundial de Suelos y han recibido poca atención preferente de especialistas en fertilidad de suelos y de economistas, debido a la creciente necesidad de utilizar con mayor eficiencia los fertilizantes, al incremento de sus precios y al imperativo de conservar el ambiente. En relación a lo anterior, Cabrera et al. (2010) determinaron que entre el $30 \%$ y $50 \%$ del rendimiento de los cultivos cañeros es atribuible a los nutrientes aplicados debido a que debe incrementarse la fertilización nitrogenada con el número de cortes (socas y resocas), ya que el vigor de las plantaciones disminuye, debido a que surgen otros problemas como el nivel nutricional, la compactación del suelo y presencia de plagas y enfermedad (Vitti et al., 2006). Por lo que para aumentar la producción de azúcar se requiere conocer la ubicación espacial de los suelos, sus propiedades físicas, químicas y biológicas, así como los procesos pedogenéticos. Así, para un agricultor cañero, un buen suelo es aquel que le permitirá producir 100 a 150 toneladas de caña por hectárea por año (Moore, 2009; Galdámez, 2007).

El diagnóstico espacial, temporal y socioeconómico de la zona cañera de la Huasteca se realizó mediante el uso de cartografía de base edafológica escala 1:50,000 del Instituto Nacional de Estadística Geografía e Informática de México (INEGI) que cubren el área de estudio (varias fechas), los datos de crecimiento cañero reportados en el Manual Azucarero Mexicano de 1958 a 2011, el análisis multiespectral y multitemporal de imágenes satelitales de 1973, 1990, 2005 y 2008 (imágenes del satélite Landsat MSS, Landsat 7 EMT+: 2645, 2745 y 2744 (órbita/ punto) y SPOT HVR 2008: 585-305, 586-305, 588-305, 587-306, 588-306, 589-306, 587- 
$307,588-307,589-307,588-308$ y $589-308$ empleando el Datum Norteamericano de 1927 y la proyección Transversal de Mercator (UTM zona 14 Norte) para Landsat y Esferoide WGS84 para SPOT para obtener los polígonos cañeros que establecen el crecimiento espacial en cuatro décadas de los municipios productores de caña de azúcar de acuerdo a la metodología de Rudorff et al., (2010) mediante el software ILWIS 3.3 (Integrated Land and Water Information System, ITC, System).

Para el nivel de aptitud se empleó la metodología de Evaluación de Tierras de FAO (FAO, 1990, 1985; los estudios de Pellegrino, 2012; Fernández et al., 2004; Andrade et al., 2010; Jiménez et al., 2004; Viloria et al., 2003; Sánchez et al., 2002; y Sellamuthu et al., 2000) de Evaluación Multicriterio en un ambiente SIG para la construcción del mapa de variabilidad de las propiedades del suelo y los criterios relevantes para delimitar las áreas adecuadas para los cultivos de caña de azúcar de Silva Cruz et al., 2011; Ruiz et al., 2011; Delgado-Caballero, 2011; CeballosSilva et al., 2010 y Bastidas-Obando, 2010, con los datos de los requerimientos edáficos de fertilidad del cultivo por grado de aptitud diferenciados (nitrógeno, fósforo, potasio, calcio, magnesio, hierro, cobre, zinc, manganeso, pH del suelo, materia orgánica, sodio, acidez intercambiable y carbonato de calcio), pendientes del terreno (\%) y de la altitud (m), de las cuatro zonas cañeras de los ingenios azucareros basados en el muestreo georreferenciado de suelos de acuerdo con la "Norma Oficial Mexicana que Establece las Especificaciones de Fertilidad, Salinidad y Clasificación de Suelos. Estudios, Muestreo y Análisis" (NOM-021-RECNAT-2000) (Cuadro $N^{\circ} 1$ ), reportado por el Servicio de Información Agroalimentaria y Pesquera (SIAP) (SIAP;

Cuadro $\mathrm{N}^{\circ} 1$

Niveles de aptitud para caña de azúcar

\begin{tabular}{|c|c|c|c|c|}
\hline Propiedad/Aptitud & Alta & Media & Baja & No Apta \\
\hline Rendimiento potencial (t/ha) & $>80$ & $80-55$ & $55-40$ & $<40$ \\
\hline Altitud & Hasta 400 & $400-850$ & $850-1,300$ & $>1300$ \\
\hline Pendiente & $0-8$ & $8-16$ & $16-30$ & $>30$ \\
\hline $\mathrm{pH}$ & $6.6-7.3$ & $\begin{array}{r}6.1-6.5,7.4 \\
-8.3\end{array}$ & $5.6-6.0,>8.3$ & $<5.5$ \\
\hline $\mathrm{CIC}$ (meq/100g) & $>20$ & $15-20$ & $15-10$ & $<10$ \\
\hline Materia orgánica (\%) & $>5$ & $3-5$ & $2-3$ & $1-2$ \\
\hline $\mathrm{N}(\%)$ & $>0.4$ & $0.1-0.4$ & $0.032-0.1$ & $<0.032$ \\
\hline$P(p p m)$ & $>40$ & $39-18$ & $17-9$ & $<9$ \\
\hline K ppm & $>468$ & $468-82$ & $82-42.9$ & $<39$ \\
\hline Ca (ppm) & $>2.004$ & $1.002-2.004$ & $400-1.002$ & $<400$ \\
\hline Mg (ppm) & $>365$ & $158-365$ & $60-158$ & $<60$ \\
\hline $\mathrm{Cu}(\mathrm{ppm})$ & $1.2-2.5$ & $0.8-1.20$ & $0.3-0.8$ & $<0.3$ \\
\hline Fe (ppm) & $16-25$ & $10-16$ & $5-10$ & $<5.0$ \\
\hline Mn (ppm) & $29-50$ & $14-29$ & $5-14$ & $<5.0$ \\
\hline Zn (ppm) & $5-8$ & $3-5$ & $1-3$ & $<1.0$ \\
\hline $\mathrm{Na}(\mathrm{ppm})$ & $<345$ & $345-575$ & $575-920$ & $>920$ \\
\hline
\end{tabular}

Fuente: Quintero, 2008; Ortega, 2007; Hunsigi, 2001; NOM, 2000; Chávez, 1999; Vázquez 1997; Humbert, 1974 
2010) y los ingenios azucareros dentro del módulo multicriterio del software ESRI ArcGis 9.2. Así se elaboraron los mapas temáticos correspondientes para visualizar la variación espacial de los atributos de fertilidad estudiados. La integración del mapa de variables edáficas constituye la zonificación edafológica del cultivo de caña en la Huasteca potosina, como resultado del efecto que ha tenido el manejo agrícola sostenido por más de 40 años.

\section{Aptitud edafológica al cultivo de caña de azúcar en la Huasteca potosina}

En la Huasteca potosina, la caña de azúcar, al igual que el resto de las plantas, requiere elementos esenciales para completar exitosamente su desarrollo fenológico (macronutrimentos y micronutrimentos), debido a su elevada capacidad de producción de material vegetal (tallos, follaje, cepas y raíces) y a la prolongada duración de su ciclo (12 a 24 meses). Un elemento esencial de fertilidad es aquel cuya ausencia causa la alteración en el desarrollo normal de la planta, según el papel que cumpla a nivel fisiológico, y altera en mayor o menor grado el rendimiento (industrial o agrícola).

En este sentido, del total de la superficie cañera evaluada $(83,689$ ha), la mayor distribución espacial (Figura 3 y Cuadro 2) presenta aptitud al cultivo de caña y se localiza principalmente en zonas de reciente incorporación (década de 1980 y 1990) al cultivo en los municipios de El Naranjo (San Miguel), Ciudad Valles (Plan de San Luis) y

Figura $\mathrm{N}^{\circ} 3$

Aptitud edafológica al cultivo de caña de azúcar en la Huasteca potosina

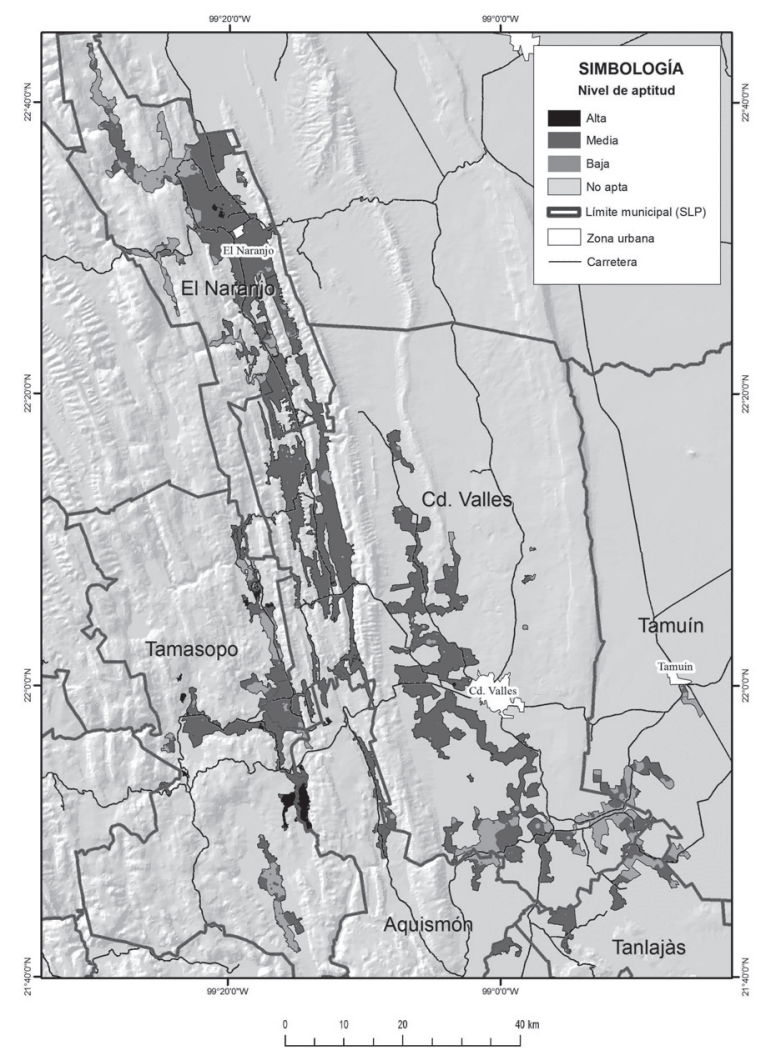

Fuente: Elaboración propia. 
EVAluación de aptitud de tierRas al Cultivo de caña de aZúCar en la

Cuadro $\mathrm{N}^{\circ} 2$

Aptitud edafológica al cultivo de caña de azúcar de los ingenios azucareros en la Huasteca potosina

\begin{tabular}{|l|r|r|r|r|r|r|r|}
\hline \multirow{2}{*}{ Productividad } & \multirow{2}{*}{$\begin{array}{c}\text { Superficie } \\
\text { (ha) }\end{array}$} & \multicolumn{2}{|c|}{ Alta } & \multicolumn{2}{c|}{ Media a baja } & \multicolumn{2}{c|}{ Muy Baja } \\
\cline { 5 - 8 } Ingenio & \multicolumn{1}{c|}{ ha } & \multicolumn{1}{c}{$\%$} & \multicolumn{1}{c|}{ ha } & \multicolumn{1}{c|}{$\%$} & \multicolumn{1}{c|}{ ha } & $\%$ \\
\hline Alianza Popular & 16.751 & $1.601,4$ & 9,56 & $12.231,6$ & 73,02 & $2.916,4$ & 17,41 \\
\hline Plan de Ayala & 30.949 & 37,14 & 0,12 & $24.007,14$ & 77,57 & $6.904,7$ & 22,31 \\
\hline Plan de San Luis & 17.228 & 18,95 & 0,11 & $16.220,2$ & 94,15 & 988,9 & 5,74 \\
\hline San Miguel & 18.761 & 983,1 & 5,24 & $9.519,4$ & 50,74 & $8.258,6$ & 44,02 \\
\hline Total & 83.689 & $2.640,59$ & 3,16 & $61.978,34$ & 74,06 & $19.068,6$ & 22,79 \\
\hline
\end{tabular}

Fuente: Elaboración propia.

Figura $N^{\circ} 4$

Crecimiento espacial de las zonas cañeras y municipios de la Huasteca

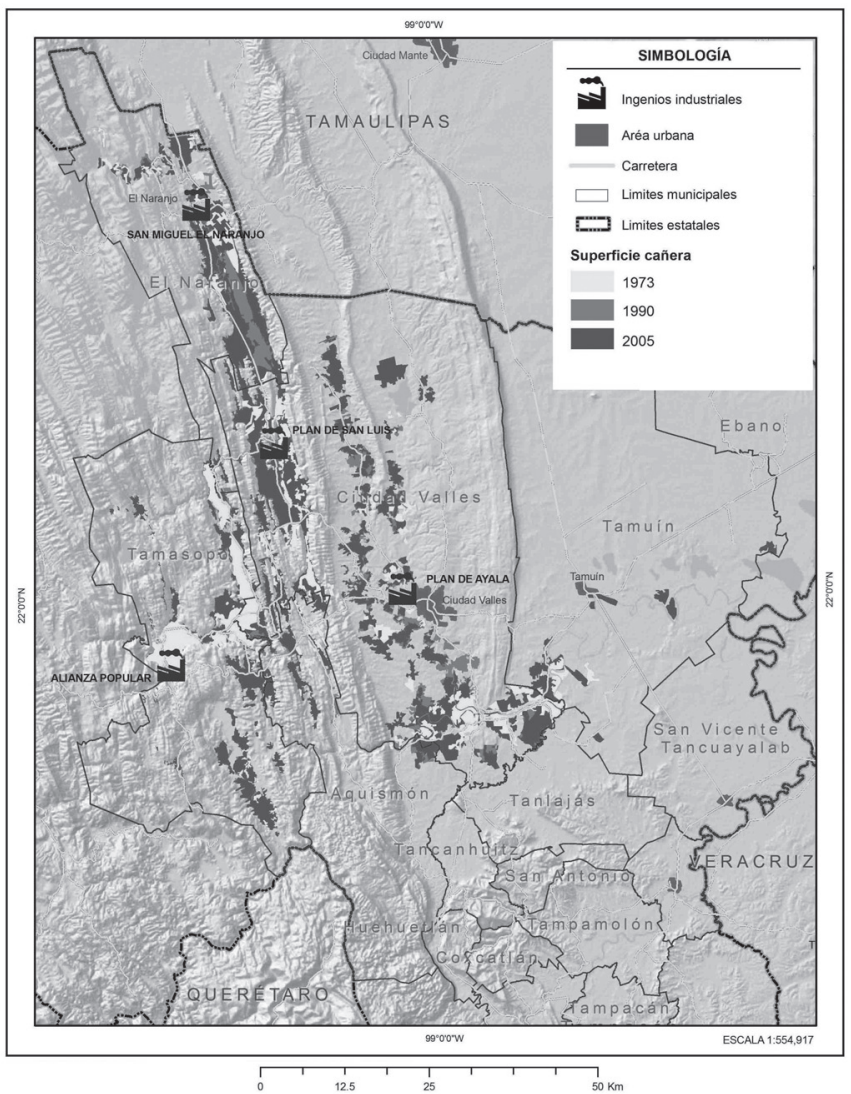

Fuente: Elaboración propia. 
Cuadro $\mathrm{N}^{\circ} 3$

Crecimiento de la superficie cañera 1970 a 2010

\begin{tabular}{|l|r|r|r|r|r|}
\hline \multicolumn{1}{|c|}{$\begin{array}{c}\text { Superficie Total } \\
\text { (ha) }\end{array}$} & \multicolumn{1}{|c|}{ Año } & $\begin{array}{c}\text { Municipio } \\
\text { Productor }\end{array}$ & \multicolumn{1}{c|}{1973} & \multicolumn{1}{c|}{1990} \\
\hline 26.438 & 1973 & Tamasopo & 8.152 & 11.774 & 18.881 \\
\hline 78.126 & 1990 & CD Valles & 13.308 & 41.712 & 38.391 \\
\hline 83.689 & 2010 & Naranjo & 2.858 & 18.662 & 26.838 \\
\hline
\end{tabular}

Fuente: Elaboración propia.

Tamasopo (Alianza Popular), y el resto corresponde a zonas más antiguas (década de 1960 y anteriores al siglo XX) en Tamasopo (Agua Buena) y Ciudad Valles (Plan de Ayala), cada una localizada alrededor de un polo o un eje (ingenio azucarero) formando un patrón espacial donde se concentraron los productores. Es decir, la Huasteca Norte y Centro son las áreas que registran el mayor incremento en su superficie dedicada al cultivo al transformarse en áreas de influencia o abasto de los ingenios (Figuras $\mathrm{N}^{\circ} 1$ y $\mathrm{N}^{\circ} 2$ 2). Este proceso comenzó desde los sectores de tierras fértiles y agricultores con tradición agrícola, y avanzó gradualmente hacia los sectores más marginales para dicha explotación (Figura $N^{\circ}$ 4 y Cuadro $N^{\circ} 3$ ).

El análisis espacial estableció que más del $74 \%$ de la superficie evaluada $(83,689$ ha $)$ permite el uso agrícola cañero de forma sostenida pero con limitaciones. De ella, solamente $2,640.59$ ha $(3.16 \%)$ presenta la clase "apta o alta», mientras que un 22,8 \% pertenece a la clase «marginalmente apta o muy baja». Este resultado es consistente con Astier et al. (2002) y Meyer y Wood (2001) quienes indicaron que un suelo fértil debe definirse como aquel que conserva las propiedades físicas, químicas y biológicas deseables, a lo largo del tiempo de uso agrícola, mientras abastece adecuadamente a la planta de caña de azúcar de agua y nutrimentos, además de proveerle de sostén mecánico. Es decir, un suelo fértil es aquel cuya producción cañera sin fertilización es elevada o produce un incremento rentable del rendimiento con una dosis relativamente baja; en cambio un suelo pobre, es aquel en el cual la no fertilización implica obtener una muy baja producción particularmente en cañas soca y resoca (ratoon).

Asimismo, SIAP (2010) reportó que esta región productora está configurada en su mayoría por zonas montañosas y cerriles; las partes planas se limitan a valles intermontanos y planicies. El tipo de suelo que predomina es de espesor variable y tiene permeabilidad moderada estable, con altos contenidos de arcilla, color rojizo a café rojizo, ricos en cuarzo y óxidos de hierro y son del grupo Vertisoles. Estos suelos durante el estiaje son duros, y presentan grietas anchas y profundas, con colores variados entre negro, rojizo y gris. Tienen una capa superficial en materia orgánica, son profundos y su susceptibilidad a la erosión es moderada.

En el área de abasto de los ingenios todavía se encuentran suelos con suficiente cantidad de materia orgánica (3,0 a 5,0 \%) considerados ricos (Figura 5), lo cual favorece una serie de condiciones físicas apropiadas para la producción de caña de azúcar (Ribón Carrillo et al., 2003); pero si no se tiene el cuidado de preservarla a través de prácticas de manejo como aportes de residuos de cosecha, compostas, vinazas y biofertilizantes entre otros (Quiroz et al., 2011) dicha condición se perderá paulatinamente causando problemas de compactación, aireación y disminución de la productividad, lo cual puede ya estar ocurriendo en varios predios de la zona, donde se acentúan problemas de baja capacidad de almacenamiento de agua y baja productividad en general, sobre todo en los ingenios azucareros Plan de Ayala y San Miguel del Naranjo. 
Figura $\mathrm{N}^{\circ} 5$

Materia orgánica en la Huasteca potosina

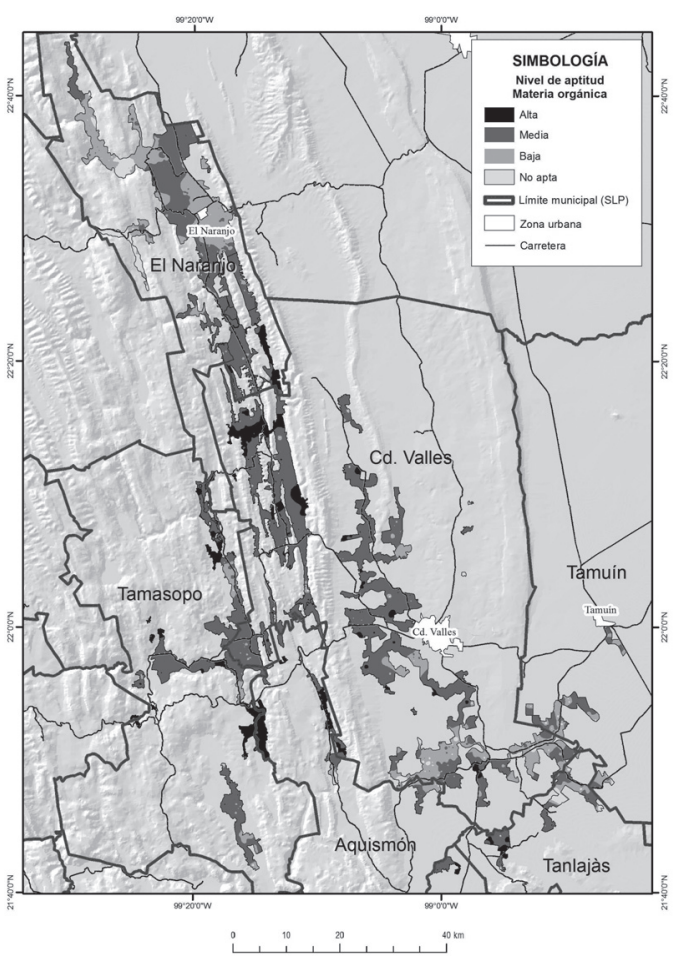

Fuente: Elaboración propia.

El contenido de materia orgánica según Faye-Savario (2011); Cabrera et al. (2010); Naranjo et al. (2006) y Ribón Carrillo et al. (2003) presenta una tendencia a disminuir con el paso de los años de monocultivo de la caña de azúcar; es decir, después de desmontar una selva, el contenido de $\mathrm{MO}$ (entre $6 \%$ y $10 \%$ ) desciende en los primeros años de 25 a $50 \%$, como el caso de estudio, esta reducción se hace más lenta y progresiva durante el tiempo del cultivo, hasta alcanzar un valor estable después de 15 años (3\% y $4 \%$ ). García-Silva et al. (2006) concluyeron que el uso antrópico del suelo en especial por monocultivos como la caña de azúcar, es el principal responsable de la reducción de sus reservas orgánicas; se han estimado pérdidas entre $30 \%$ y $50 \%$ del carbono orgánico edáfico (COS) en la capa arable, después de 20 a 30 años de cultivo

Sin embargo, las fuentes de MO pueden disminuir a una alta velocidad por las prác- ticas de eliminar los residuos de la cosecha (paja, tlazole, puntas de caña y cogollos) mediante quema y requema $(90 \%$ de la zona de estudio la emplea como práctica convencional), ya que los residuos pueden ser oxidados más rápidamente debido al aumento de la temperatura por efecto de la quema de la caña de azúcar antes de su cosecha, posteriormente, el contenido de $\mathrm{MO}$ del suelo tiende a estabilizarse hasta los 20 años de cultivo, pero sin alcanzar su nivel inicial, registrándose a los 30 años de cultivo una reducción en su contenido por la quema más que a las labores culturales del mismo y el monocultivo (Ribón Carrillo et al., 2003). Lo anterior demuestra que la $\mathrm{MO}$ es un índice confiable para evaluar la fertilidad y/o degradación del suelo, retener la humedad y conservar la estructura del suelo y está estrechamente relacionada con el tiempo de cultivo con caña de azúcar (Armida-Alcudia et al., 2005). 
Para Salgado et al. (2001) la quema de 1 ha de caña de azúcar libera a la atmósfera 6.6 Mg de C al año, equivalente a 24.3 Mg de $\mathrm{CO}_{2}$, planteándose, además, que esta última cifra, comparada con la capacidad de fijación de $\mathrm{CO}_{2}$ por este cultivo, no resulta significativa, ya que 1 ha de caña de azúcar con alta tasa de crecimiento es capaz de secuestrar $80 \mathrm{Mg}$ de $\mathrm{CO}_{2}$ anualmente, lo que equivale a 21.7 Mg.año-1 de C. Sin embargo, Herrera et al. (2009) y Milanes et al. (2000) lograron demostrar que la quema de la caña de azúcar antes de la cosecha puede llegar a producir pérdidas de nitrógeno hasta de $24 \mathrm{~kg} / \mathrm{ha}$ en la quema y 17 kg/ha en la requema, en dependencia de la variedad utilizada, ciclo de cosecha y condiciones de cultivo

Por lo tanto, la disminución de las reservas orgánicas del suelo trae como consecuencia, a corto plazo, una reducción del rendimiento potencial de los cultivos, una baja actividad biológica, y a mediano plazo, el suelo se hace vulnerable a la compactación, le origina una deficiente estructuración, lo que causa un inadecuado drenaje y una menor capacidad de retención hídrica y se reduce la capacidad de restauración de nutrimentos, exponiendo el sistema a la acción erosiva (Aparecida Souza et al., 2012; Quiroz et al., 2011; García-Silva et al., 2006). Esto evidencia la necesidad de nuevos sistema de cosecha y recolección de la materia prima y evitar la quema de caña e incorporar los residuos de cosecha.

De este modo, para la zona de estudio, carecer de un programa de gestión, planeación y control sistemático de procedimientos de operación en el campo cañero, implica el fracaso de cualquier esfuerzo tendiente a fomentar la sustentabilidad de los procesos agroproductivos y de la transformación de la caña de azúcar. En consecuencia, para promover ingenios azucareros competitivos y diversificados, se requiere la reordenación de la superficie cañera sembrada en sitios donde las condiciones agroecológicas garanticen la productividad creciente.

\section{Conclusiones}

Aunque esta región posee condiciones edafológicas apropiadas para plantaciones cañeras en el $74 \%$ de la superficie, la localización de los mejores lugares para su establecimiento es una estrategia necesaria para dirigir acciones de manejo diferenciado, intensificación agrícola y conservación de suelos (compostas, cosecha en verde, enmiendas orgánicas, biofertilizantes, rotación de cultivos, etc.) para maximizar probabilidades de éxito (incremento de rendimientos y productividad), con lo que se visualizara la expectativa de los beneficios económicos y disminuyen los riesgos del propietario.

Si bien hasta este punto se confirmó la eficiencia de las metodologías tradicionales de muestreo y la aplicación de la estadística tradicional con base en la fertilidad para este cultivo, estas no son capaces de ofrecer una visión espacial de esta variación, pues se parte del supuesto que toda el área muestreada posee el mismo valor promedio para las variables de interés, lo cual no es del todo cierto.

Al Ilevar a cabo el análisis espacial se encontró que las variables de fertilidad, evaluadas para los suelos cañeros están autocorrelacionadas, y que en el espacio se establecen gradientes para cada una de las variables de fertilidad estudiadas. Es decir, los mapas de fertilidad representan espacial y visualmente la zonificación edafológica cañera, con la diferencia de que permiten detectar en cada una de las áreas, no un valor único de fertilidad, sino gradientes de concentración que representarían el comportamiento que ocurriría normalmente en la naturaleza. Lo anterior sugiere un manejo a nivel de sitio específico e incluso a nivel de áreas por ingenio y dentro de cada lote, identificando zonas con limitaciones importantes que afecten al rendimiento y la productividad. Se concluye que la distribución espacial, de las características químicas asociadas con la fertilidad del suelo en la Huasteca, está fuertemente ligada al manejo agronómico de los últimos 40 años y que las herramientas geomáticas empleadas demostraron ser útiles para evaluar el área cañera para su uso en la agricultura de precisión en el corto plazo.

\section{Referencias bibliográficas}

AGUILAR, R.N.; GALINDO, M.G. y FORTANELLI, M.J. Zonificación productiva cañera 
en la Huasteca Potosina México. Revista Agronomía Tropical Venezuela Agronomía Tropical, 2010, Vol. 60, N², p. 139-154.

AGUILAR, R. N.; RODRÍGUEZ, L.; ENRÍQUEZ, R.; CASTILLO, M. \& HERRERA, S. The Mexican sugarcane industry, overview, constraints, current status and long-term trends. Springer Sugar Tech, 2012, Vol. 14, N³ p. 207-222.

ANDRADE, O.; BRICEÑO, S; ERASMI, M; KAPPAS, J y UNDA, E. Generación y mapeo de parámetros ambientales con fines de evaluación de tierras en el municipio Torres, Estado Lara, Venezuela. Bioagro, 2010, Vol. $22 \mathrm{~N}^{\circ} 2$ p. $115-126$.

APARECIDA-SOUZA, A.; SANTOS, T.; MACHADO, W.; HUNGRIA, M.; TAVARES, J. \& GUIMARAES, M.F. Effects of sugarcane harvesting with burning on the chemical and microbiological properties of the soil. Agriculture, Ecosystems and Environment, 2012, Vol. 155 p.1-6.

ARMIDA-ALCUDIA, L.; ESPINOSA, D. \& PALMA, J. Carbono en biomasa microbiana y carbono soluble como indicadores de calidad de vertisoles cultivados con caña azucarera. Terra Latinoamericana, 2005, Vol. $23 \mathrm{~N}^{\circ}$. 4, p. 545-551.

ASTIER, C.; MASS, M. ETCHEVERS, B. Derivación de indicadores de calidad de suelos en el contexto de la agricultura sustentable. Agrociencia, 2002, Vol. 36, p. 605-620.

BARBOSA QUEIROZ, R.; MATHIAS DOS SANTOS, D. M.; FERRAUDO, S.; DOMINGUES CARLIN, S. \& ALMEIDA SILVA, M. Biochemical and physiological responses of sugarcane cultivars to soil water deficiencies. Scientia Agricola (Piracicaba, Braz.), 2011, Vol. 68, N4, p. 469-476.

BASTIDAS-OBANDO, E. y CARBONELL, J. A. Caracterización espectral y mineralógica de los suelos del valle del río Cauca por espectroscopía visible e infrarroja (400 - 2.500 nm). Agronomía Colombiana, 2010, Vol. XXVIII, No 2, p. 291-301.

CABRERA, J. A. y ZUAZNÁBAR, R. Impacto sobre el ambiente del monocultivo de la caña de azúcar con el uso de la quema para la cosecha y la fertilización nitrogenada. I. Balance del carbono. Cultivos Tropicales, 2010a, Vol. 31, Nº 1 p. 5-13.

CABRERA, J. A. y ZUAZNÁBAR, R. Respuesta de la caña de azúcar a la fertilización nitrogenada en un experimento de larga duración con 24 cosechas acumuladas. Cultivos Tropicales, 2010b, Vol. 31, №1, p. 93-100.

CEBALLOS-SILVA, A. P. y LÓPEZ-BLAN$C O$, J. Delimitación de áreas adecuadas para cultivos de alternativa: una evaluación multicriterio-SIG. Terra latinoamericana, 2010, Vol. 28, $\mathrm{N}^{\circ} 2$, p. 109-118.

CHÁVEZ, M. Nutrición y fertilización de la caña de azúcar en Costa Rica. XI Congreso Nacional Agronómico y III Congreso Nacional de Suelos, 1999, p.193-214

CHUVIECO, E. Fundamentos de teledetección espacial. Madrid: Ediciones RIALP, 1996.

CÁMARA NACIONAL DE LAS INDUSTRIAS AZUCARERA Y ALCOHOLERA (CNIAA). Manual Azucarero Mexicano 2011. México: Cámara Nacional de las Industrias Azucarera y Alcoholera, 2011.

COTLER H. El uso de la información edáfica en los estudios ambientales, 2003. Disponible en Internet: http://www2.ine.gob.mx/ publicaciones/gacetas/399/cotler2.htm

DELGADO CABALLERO, E. C.; VALDEZ LAZALDE, J. R.; FIERROS, A. M.; DE LOS SANTOS, P. y GÓMEZ, G. Aptitud de áreas para plantaciones de eucalipto en Oaxaca y Veracruz: proceso de análisis jerarquizado vs. Álgebra booleana. Revista Mexicana de Ciencias Forestales, 2011, Vol. 1. Nº 1, 124-133.

ORGANIZACIÓN DE LAS NACIONES UNIDAS PARA LA ALIMENTACIÓN Y LA AGRICULTURA (FAO). Directivas: Evaluación de tierras para la agricultura en secano. Boletín de Suelos, 1985, N 52, p. 228.

ORGANIZACIÓN DE LAS NACIONES UNIDAS PARA LA ALIMENTACIÓN Y LA AGRICULTURA (FAO). Evaluación de tierras 
para la agricultura en regadío: directivas. Boletín de suelos, 1990, № 55, p. 289.

FAYE SAVARIO C. \& HOY, J. W. Microbial communities in sugarcane field soils with and without a sugarcane cropping history. Plant Soil, 2011, Vol. 341, p. 63-73.

FERNÁNDEZ, O.R.; PÉREZ, R. \& GUBINELLI, A. Effect of soil spatial variability on sugarcane parameters (Saccharum officinarum) in Monay, Trujillo State. Venesuelos, 2004, Vol. 12 , p. 30-43.

GALDÁMEZ, G. J. Manejo sostenible de suelos en la producción agrícola de la depresión central de Chiapas, México. I seminario de cooperación y desarrollo en espacios rurales iberoamericanos, sostenibilidad e indicadores. Almería, 16-17 octubre 2007.

GALINDO, M. G.; CONTRERAS, C. y OLVERA, S.L. Metodología para determinar zonas de peligro al ataque de la langosta centroamericana (Schistocerca piceifrons piceifrons Walker) apoyados en sensores de alta resolución y SIG. Estudio de caso la Huasteca México. En: AGUIRRE GÓMEZ, R. (compilador). Conceptos de geomática y estudios de caso en México. México D.F.: UNAM-Ig, Serie libros de investigación, 2009, № 5.

GARCÍA-SILVA, R.; ESPINOSA-VICTORIA, D.; FIGUEROA-SANDOVAL, B.; GARCÍACALDERÓN, N.E. \& GALLARDO-LANCHO, J.F. Soil Organic Carbon and Humic Fraction Stocks in a Vertisol under Non-tillage Management. Terra Latinoamericana, 2006, Vol. 24, $\mathrm{N}^{\circ} 2$ p. 241-251.

GHIBERTO, P.J.; LIBARDI, P.L.; BRITO, A.S.; TRIVELIN, P.C.O. Components of the water balance in soil with sugarcane crops. Agricultural Water Management, 2011, Vol. 102, p.1-7.

GÓMEZ, I.A.; HERNÁNDEZ, A.; ASCANIO, M. O.; SÁNCHEZ, M. E.; DURÁN, J. L.; BENÍTEZ, LEDYA; VILLEGAS, R.; PONCE DE LEÓN, D. y LÓPEZ, M. Grupos de suelos y su distribución en las áreas del Ingenio Central Motzorongo, Veracruz, México. Cultivos Tropicales, 2010, Vol. 31, Nº 2 p. 32-36.
HENRÍQUEZ, C.; KILLORN, R.; BERTSCH, F. y SANCHO, F. La geoestadística en el estudio de la variación espacial de la fertilidad del suelo mediante el uso del interpolador Kriging. Agronomía Costarricense, 2005, Vol. 29, p. 73-81.

HERRERA SOLANO, A.; MILANÉS RAMOS, N.; MOLINA LARA, F.; ORDÓÑEZ, P.; BARAHONA, P.; ELORZA MARTÍNEZ, A.; CASTILLO MORÁN, V.; ENRÍQUEZ RUVALCABA, D.A. \& RODRÍGUEZ, L. Effect of management of the harvest wastes of sugar cane (Saccharum spp. hybrid) on the field performance in Veracruz, México. Revista UDO Agrícola, 2009, Vol. $9 \mathrm{~N}^{\circ}$ 3, p. 517 521.

HUANG, X.; GRACE, P.; MENGERSEN, K. \& WEIER, K. Spatio-temporal variation in soil derived nitrous oxide emissions under sugarcane. Science of the Total Environment, 2011, Vol. 409, p. 4572-4578.

HUMBERT, R. P. El cultivo de la caña de azúcar. México D.F.: Editorial CECSA, 1974.

HUNSIGI, G. Sugarcane in Agriculture and industry. Bangalore: Eastern Press Pvt Ltd., 2001.

JIANG, Z.P.; YANG, R.L.; GUANG-PO, W.; QING, L.; TIAN-MING, S.; YAN-CHENG, M.; HAI-YING, Z. \& CHANG-YAN, L. Effect of Long-Term Vinasse Application on Physico-chemical Properties of Sugarcane Field Soils. Sugar Tech, 2012. Disponible en internet: http://link.springer.com/article/10.1007/ s12355-012-0174-9/fulltext.html

JIMÉNEZ, C.A.; VARGAS, V.; SALINAS, W.E.; AGUIRRE, M. y RODRÍGUEZ, D. Aptitud agroecológica para el cultivo de la caña de azúcar en el sur de Tamaulipas, México. Investigaciones Geográficas, 2004, № 53, p. 58-74.

LUNA GONZÁLEZ, C.A.; COCK, J.H.; PALMA, A.E.; DIAZ, L.V. y MORENO, C.A. Análisis de la productividad en la agroindustria azucarera de Colombia y perspectivas para aumentarla. En: CENICAÑA. El cultivo de la caña en la zona azucarera de Colombia. Cali: CENICAÑA, 1995, p. 373-394. 
MARQUES-GENÚ, A. \& MELO-DEMATTÊ, J.A. Prediction of soil chemical attributes using optical remote sensing. Acta Scientiarum Agronomy, 2011, Vol. 33, N4, p. 723-727.

MEYER, J.H \& WOOD, R.A. The effects of soil fertility and nutrition on sugarcane quality: a review. Proc SAfr Sug Technol Ass, 2001, $N^{\circ} 75$ p. 242-247.

MILANÉS, R.N.; LOZANO, L.F. y ORDÓÑEZ, B.P. Efectos de la quema y extracción de nutrimentos por la caña de azúcar y las malezas en la Región Veracruz Central. Memorias, 2000, 30 Congreso de la Sociedad Mexicana de la Ciencia del Suelo, Veracruz, México.

MIPHOKASAP, P.; HONDA, K.; VAIPHASA, C.; SOURIS, M. \& NAGAI, M. Estimating Canopy Nitrogen Concentration in Sugarcane Using Field Imaging Spectroscopy. Remote Sensing, 2012, Vol. 4, p.1651-1670.

MOORE, P.H. Sugarcane Biology, Yield, and Potential for Improvement. Workshop BIOEN on Sugarcane Improvement, 18 e 19 de março, São Paulo, 2009. Disponible en Internet:

http://www.fapesp.br/materia/5064/bioen/ workshop-bioen-on-sugarcane-improvement18-e-19-3-2009-.htm

NARANJO, J.S.; SALGADO-GARCÍA, L.C.; LAGUNES-ESPINOZA, E.; CARRILLO-AVILA, D. \& PALMA-LÓPEZ, J. Changes in the properties of a Mexican Fluvisol following 30 years of sugarcane cultivation. Soil and Tillage Research, 2006, Vol. 88, N 1-2, p. 160-167.

NOM 2000. Norma Oficial Mexicana que Establece las Especificaciones de Fertilidad, Salinidad y Clasificación de Suelos. México: Estudios, Muestreo y Análisis. NOM021-RECNAT-2000, Secretaría de Medio Ambiente y Recursos Naturales.

ORTEGA, A. Diagnóstico de suelos. Instituto Nacional de Tecnología Agropecuaria Estación Experimental Agropecuaria Salta. Disponible en Internet:

http://www.inta.gov.ar/salta/info/documentos/Suelos/DIAGNOSTICO\%20SUELOS. pdf.
PALANISWAMI, C.; GOPALASUNDARAM, P. \& BHASKARAN, A. Application of GPS and GIS in Sugarcane Agriculture. Sugar Tech, 2011, Vol. 13, N4, p. 360-365.

PANOSSO, A.R.; J. MARQUES JR.; MILORI, D.M.B.P.; FERRAUDO, A.S.; BARBIERI, G.T.; PEREIRA, N. \& LA SCALA, JR. Soil CO2 emission and its relation to soil properties in sugarcane areas under Slash-and-burn and Green harvest. Soil \& Tillage Research, 2011, Vol. 111, p. 190-196.

PARÉ, L. El Estado, los cañeros y la industria azucarera, 1940-1980. México: UAM-A, UNAM, 1987.

PATIL, V.C.; NADAGOUDA, B.T. \& ALGAADI, K.A. Spatial Variability and Precision Nutrient Management in Sugarcane, 2012. Disponible en Internet:

http://www.springerlink.com/content/ h63l83229920w328/.

PELLEGRINO, D.G. \& GRAZIANO, P.S. Correlation of physical and chemical attributes of soil with sugarcane yield, 2012, Vol.47, No.4, p. 613-620.

PORTZ, G.; MOLIN, J.P. \& JASPER, J. Active crop sensor to detect variability of nitrogen supply and biomass on sugarcane fields. Precision Agric, 2012, Vol. 13, p. 33-44.

QUINTERO, D.R. Efectos de la aplicación de elementos menores en caña de azúcar en suelos del valle del río Cauca, 2008, p. 1825. Disponible en Internet:

http://www.tecnicana.org/pdf/2008/tec_ v12_no20_2008_p18-26.pdf.

QUIROZ, G.I.; PÉREZ, A.; LANDEROS, C.; MORALES, S. \& ZETINA, L. Percepción $y$ actitud de productores cañeros sobre la composta de cachaza y vinaza. Tropical and Subtropical Agroecosystems, 2011, Vol.14, p. 847-856.

RIBÓN CARRILLO, M.A.; SALGADO GARCÍA, S.; PALMA-LÓPEZ, D.J. y LAGUNES-ESPINOZA, L.C. Propiedades químicas y físicas de un vertisol cultivado con caña de azúcar. Interciencia, 2003, Vol. 28, № 3, p. 154-159. 
ROMERO, E.R. Manual del cañero. Tucumán: Estación Experimental Agroindustrial Obispo Colombres (EEAOC), 2009.

RUDORFF, B.F.T.; ALVES DE AGUIAR, D.; FERNANDO DA SILVA, W.; MIURA SUGAWARA, L.; ADAMI, M. \& ALVES MOREIRA, M. Studies on the Rapid Expansion of Sugarcane for Ethanol Production in São Paulo State (Brazil) Using Landsat Data. Remote Sensing, 2010, N², p. 1058-1076.

RUIZ, J.; HERNÁNDEZ-RODRÍGUEZ, G.E. \& ZULUETA-RODRÍGUEZ, R. Análisis de series de tiempo en el pronóstico de la producción de caña de azúcar. Terra latinoamericana, 2011, Vol. 29, №1, p. 103-109.

SALGADO, G. S; BUERO, L.; RIESTRA, D. y LAGUNES-ESPINOZA, L. C. Caña de azúcar, hacia un manejo sustentable. Villahermosa: Fundación Produce, 2001.

SÁNCHEZ, P.G.; ORTIZ, C.A.; GUTIÉRREZ, M.C. \& GÓMEZ, C.J. Local Land Classification and its Relationship with Sugarcane Crop in the South of Veracruz. Terra Latinoamerica, 2002, Vol. 20, N 4, p. 359- 369.

SÁNCHEZ-HERNÁNDEZ R.; RAMOS-REYES, R.; GEISSEN, V.; MENDOZA-PALACIOS, J.; DE LA CRUZ-LÁZARO, E.; SALCEDOPÉREZ, E. y PALMA-LÓPEZ, D. J. Contenido de carbono en suelos con diferentes usos agropecuarios en el trópico mexicano. Terra latinoamericana, 2011, Vol. 29, № 2, p. 211 219.

SELLAMUTHU NATARAJAN, S.; SIVASAMY, R. \& MANI, S. Delineating Soil Related Constraints in Sugarcane Growing Areas. Sugar Tech, 2000, Vol. 2, №3, p. $30-33$.
SISTEMA DE INFORMACIÓN AGROPECUARIA (SIAP). Sistema de Información Agropecuaria, 2010. Disponible en Internet: h t t p : // w w w. sia p.gob. mx/index. php?option $=$ com_content $\&$ view $=$ article $\& i d=5$ 3\&ltemid=325.

SILVA-CRUZ, J.; NONATO DE ASSIS JÚNIOR, R.; ROCHA MATIAS, S.S. \& HERNÁN CAMACHO-TAMAYO, J. Spatial variability of an Alfisol cultivated with sugarcane. Ciencia e Investigación Agraria, 2011, Vol. 38, p. 155164.

THORBURN, P.J.; MEIER, E.A.; COLLINS, K. \& ROBERTSON, F.A. Changes in soil carbon sequestration, fractionation and soil fertility in response to sugarcane residue retention are site-specific. Soil \& Tillage Research, 2012, Vol. 120, p. 99-111.

VÁZQUEZ, A. Guía para interpretar el análisis químico del suelo y agua. UACH, 1997.

VILORIA, J.A.; PINEDA, C.; LÓPEZ, D. y GABALDÓN, O. Aptitud de la tierra para caña de azúcar y banano en el sistema de riego tagua guay. (Aragua-Venezuela). Agronomía Tropical, 2003, Vol. 53, № 2. Disponible en Internet:

http://www.scielo.org.ve/scielo. php? script=sci_arttext \&pid=S0002-192X2003 000200006\&lng=es\&nrm=iso.

VITTI, A. C.; TRIVELIN, P. C.; CASTRO, G. J. y PENATTI, C. P. Productividad de la caña de azúcar relacionada al nitrógeno residual de la fertilización del suelo y al almacenamiento de $\mathrm{N}$ y $\mathrm{S}$ en el sistema radicular. En: VI Congreso de la Asociación de Técnicos Azucareros de Latinoamérica y el Caribe ATALAC, Guayaquil, 12 al 15 Septiembre de 2006. 